\title{
Experiences with the use of interrupted carmine red and continuous chromium sesquioxide marking of human faeces with reference to calcium, phosphorus, and magnesium
}

\author{
G. ALAN ROSE \\ From the Metabolic Unit, Department of Medicine, \\ The General Infirmary Leeds
}

The normal adult in calcium balance excretes some $80 \%$ of the intake in the stools. This is in contrast to the excretion of sodium, potassium, and nitrogen which is predominantly in the urine. It follows that whereas inaccuracies in measurement of faecal sodium, potassium, and nitrogen may not seriously affect overall balance results, similar inaccuracies are not permissible in calcium balance studies. Irregularities in bowel habits make 24-hour collections of stools almost meaningless in the majority of patients, and to overcome this difficulty Albright and his collaborators therefore utilized carmine red faecal markers given orally every few days so as to permit timing of the faeces (Reifenstein, Albright, and Wells, 1945). Since then, most investigators have used this principle, although occasionally different marker dyes have been used and periods have varied from three to seven days. However, this technique is subject to certain practical difficulties. First, collections of faeces are known never to be complete because of loss on toilet paper and in general manipulation. Secondly, other unknown losses may occur through accidental rejection of stools (an event which may occur even in the best regulated of metabolic units). Thirdly, the marker dye is not always clearly visible and is not always discretely localized. In addition there are theoretical objections to the use of these markers at all. Humphreys and Scott (1962) showed that nylon balls and chromium sesquioxide separated on passage through the alimentary tract of the cat. Wiggins and Dawson (1961) showed that a standard test meal, which contained phenol red, dissociated in the stomach into a solid phase rich in fat and poor in marker, and a liquid phase poor in fat and rich in marker. The liquid phase was preferentially emptied from the stomach. McSwiney (personal communi- cation) gave carmine red and charcoal markers 12 hours apart and observed them in the stools in the reverse order on each of the three occasions when this was tried.

Most investigators when discussing the use of carmine red marking of stools will admit that faecal results so obtained often vary remarkably from period to period. Several groups have attempted to improve the accuracy of faecal collections by using continuous faecal marking with chromium sesquioxide. The most extensive study in humans yet reported was by Whitby and Lang (1960) who studied 40 individuals, using three-day faecal periods without the use of intermittent dye markers. They observed no side effects from the use of the chromium sesquioxide and found that they could correct for irregularities in bowel action and for incomplete faecal collections. The apparent advantages of continuous chromium markings are impressive and this procedure was therefore adopted in this unit in January 1962. The interrupted carmine red technique offers one clear advantage however. When there is a sudden change in diet (or therapy) it is desirable to divide the stools into pools corresponding to the old and the new diets (or therapy) respectively, as otherwise the results may be 'blurred'. We have therefore used both continuous chromium and interrupted dye markers in combination.

Over 100 six-day balance periods have now been completed and the first 103 results from 21 studies on 18 patients are reported here. Examination of the results reveals for the first time to what extent the 'six-day' faecal collection of the carmine red technique varies from a true six-day faecal collection. In three patients, each individual stool was analysed for chromium, calcium, and phosphorus (results for 
fat are to be reported elsewhere), and in two of these cases random samples of each stool were also analysed for the same components. It is therefore possible to assess, first, to what extent faecal losses can be assessed by recovery of chromium, and secondly whether the small random sample is representative of the homogenized stool with regard to chromium, calcium, and phosphorus (and fat).

\section{METHODS}

All the patients were in a metabolic unit and the general principles of Reifenstein et al. (1945) were observed. Any given patient ate the same normal diet every day throughout his study period. The calcium content of this diet matched, as closely as could be ascertained by a dietitian, the patient's own normal intake of calcium. When the diet was commenced, capsules containing $0.5 \mathrm{~g}$. chromium sesquioxide $(1.025 \mathrm{~g}$. per day of chromium) were started, and given thrice daily until the study ended. These capsules were prepared and administered exactly as described by Whitby and Lang (1960) except that two children of 11 years (cases 16 and 18 of Fig. 3) were given only half doses of chromium. After five or six days of equilibration on the diet, the first carmine red marker was given at breakfast time, and this was repeated every six days until the study ended. Stools were homogenized in six-day periods as indicated by the beginning of the carmine markers, except when individual stools were analysed, when these were homogenized individually (after removal of small random samples in some cases). Homogenized and random samples of stool were dried at $100^{\circ} \mathrm{C}$. and ashed at $600^{\circ} \mathrm{C}$. for 12 to 16 hours. The chromium sesquioxide was oxidized with perchloric acid and assayed by the flame photometric method of Anderson and Weinbren (1961). Calcium was determined on the same oxidized ashings using the flame photometric technique of MacIntyre (1957) with only minor modifications. The Zeiss PMQ II spectrophotometer with flame attachment was used throughout for the calcium and chromium estimations. Phosphorus was measured in the same ashings on the Technicon AutoAnalyzer by the method of Fiske and Subbarow (1925). Magnesium was measured on a separate sample of homogenate by Dr. F. W. Heaton of the Department of Urology, Leeds General Infirmary, using atomic absorption spectrophotometry as described by Dawson and Heaton (1961).

In order to see whether results were more consistent by correcting for chromium content of the 'six-day' faecal collection, all other results were multiplied by the factor: chromium given in six days/chromium found in 'six-day' faecal collection.

\section{RESULTS}

When chromium sesquioxide was added to stools already in the homogenizer and immediately before homogenization so as to exclude losses due to

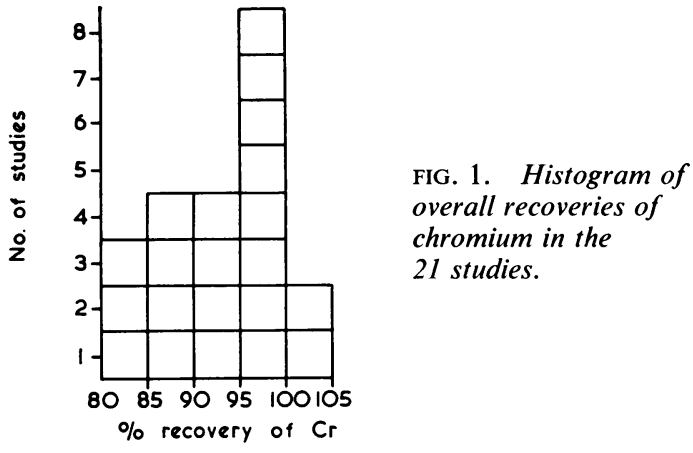

manipulation, the recoveries of chromium on four separate additions were respectively $97 \cdot 6 \%, 103 \cdot 1 \%$, $100.8 \%$, and $98.4 \%$.

The total chromium recovery in the 21 studies was $578 \mathrm{~g}$. or $93.0 \%$ of the $621 \mathrm{~g}$. administered. The minimum total recovery in any study was $80.0 \%$ and the maximum total recovery in any study was $103 \%$. The distribution of these total recoveries is shown in Figure 1.

The chromium recoveries in each of the 103 six-day faecal collections are shown in Figures 2 and 3. Figure 2 shows the overall distribution of the recoveries and Fig. 3 shows recoveries in the 21 individual studies on the 18 patients.

Figure 4 shows the faecal results from one patient both with and without correction (see above) by the

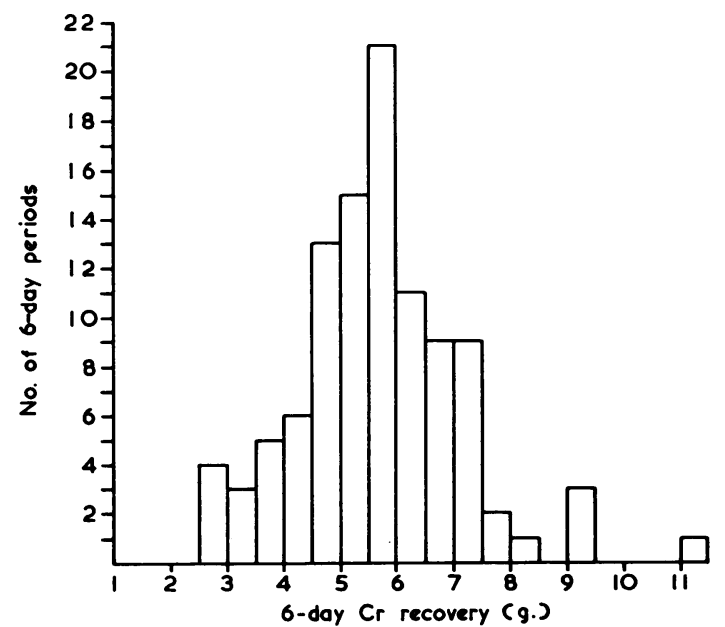

FIG. 2. Histogram of chromium recoveries in 103 consecutive six-day periods. In each six-day period $6.16 \mathrm{~g}$. of chromium was given, except that the two children (nos. 16 and 18) received half this dose. Results from these children have been multiplied by 2 to make them comparable with the other data. 


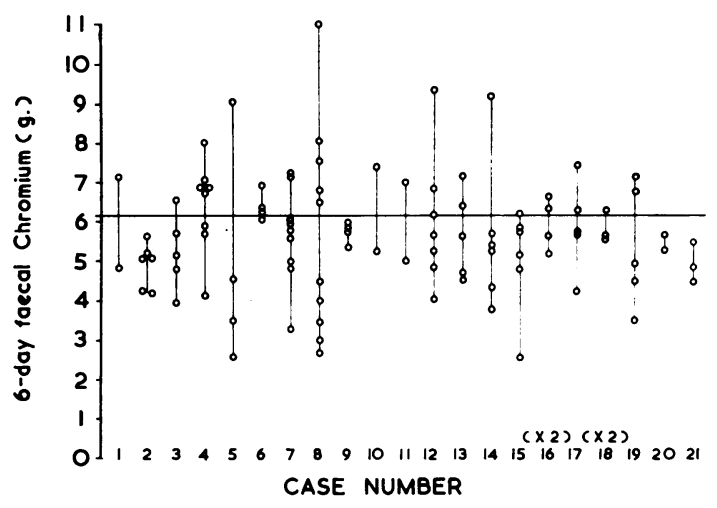

FIG. 3. The same data as in Fig. 2 but re-arranged to show the variations in chromium recoveries in each patient.

chromium method. These results are selected for illustration because this patient (no. 8) passed very constipated and dark stools so that carmine red markers were difficult to see, and the chromium contents of the 'six-day' stools were the most irregular that we encountered (see Fig. 3).

Results of individual stool analysis on patient E.Wi. are shown in Figure 5. Results of analysis of fractions of stools from patients A.R. and E.McC. are shown in Figs. 6 and 7 respectively.

\section{DISCUSSION}

The chromium sesquioxide capsules were tolerated well by the patients, whether adults or children, and no side effects have been observed. The stools were generally olive green in colour, against which background the carmine red could usually be readily identified.

Since some faeces is always lost on the toilet paper, and some is also lost in transfer from the cellophane wrapper to the homogenizer, chromium recoveries of $100 \%$ are not to be expected. The overall chromium recovery of $93 \%$ must therefore be considered satisfactory. On most patients the overall chromium recovery was 90 to $100 \%$ (see Fig. 1) but on a few patients the recovery dropped to as low as $80 \%$. In some of these cases, the low recoveries could be attributed to the short duration of the studies (12 days only in some cases) but in some other cases this explanation was not applicable and it is believed that there must have been faecal losses which would not otherwise have been recognized. Taken with the other evidence revealed below, an average loss of $7 \%$ of chromium must indicate a corresponding $7 \%$ loss of faecal calcium, phosphorus, and magnesium. This means that faecal measurements of calcium on a normal calcium intake may be 50 to $100 \mathrm{mg}$. a day too low when uncorrected for chromium. On a high
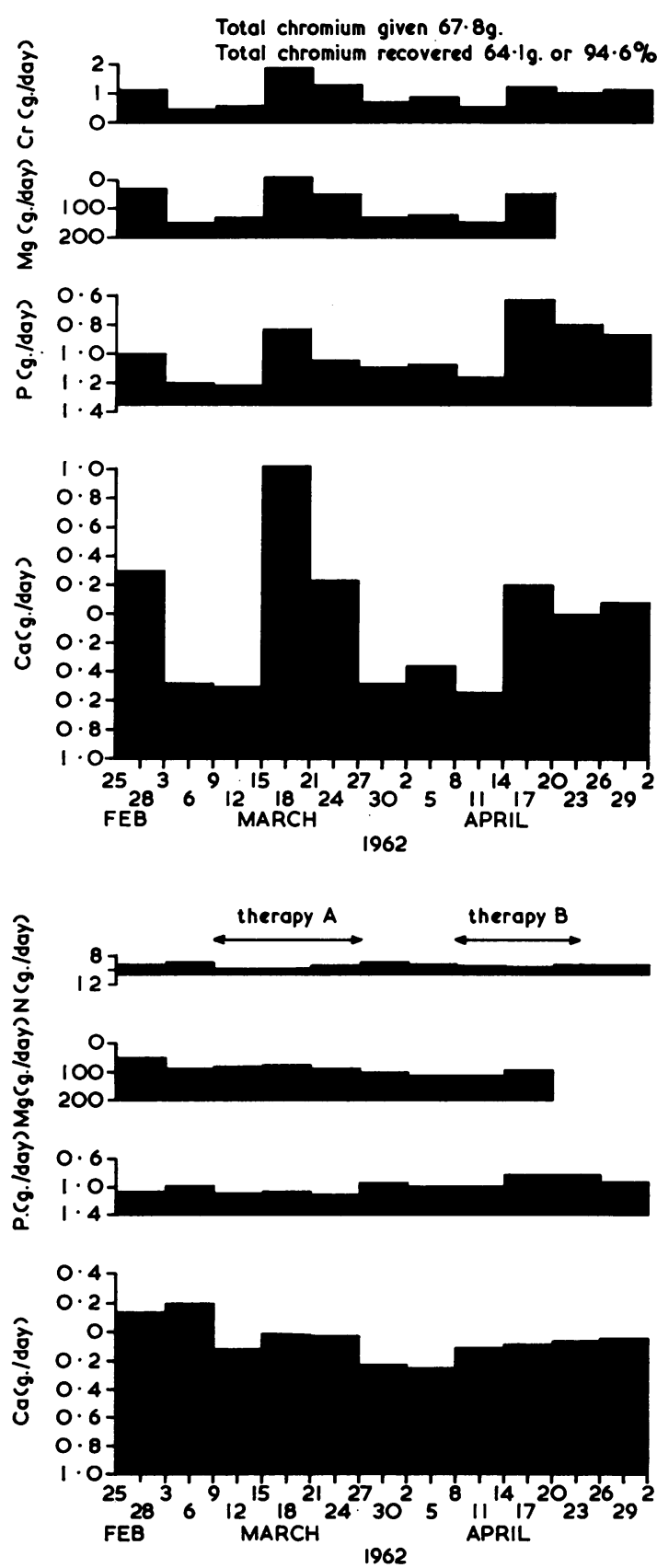

FIG. 4. Results of 'six-day' faecal collections from patient no. $8 A$ with, and $B$ without, correction for chromium recoveries. Results on this constipated patient are quite valueless when uncorrected for chromium, but when the corrections are made it is seen that changes in faecal calcium are referable to changes in therapy. Note especially the constancy of the faecal magnesium, this being uninfluenced by the therapy. 


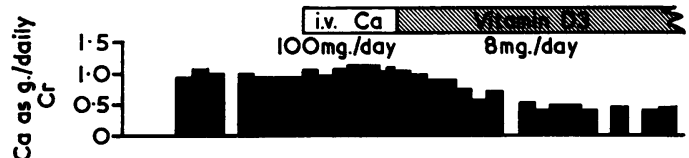

ن
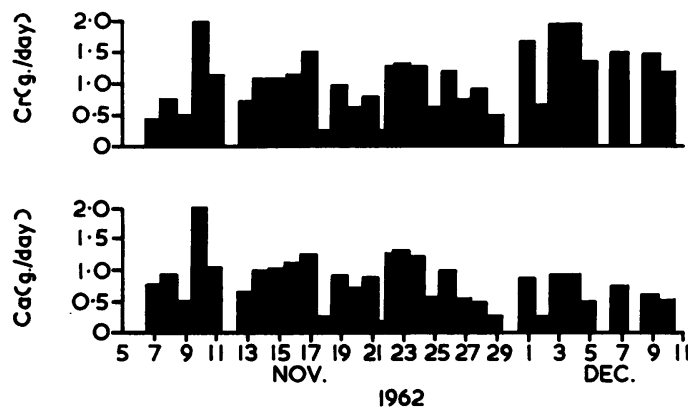

FIG. 5. Individual stool analyses on patient E.Wi. Note how during preliminary period without therapy the faecal calcium and chromium rise and fall together so that the calcium per day's chromium intake is nearly constant. The rise in faecal calcium due to the intravenous calcium therapy, although quite small, can be clearly seen.

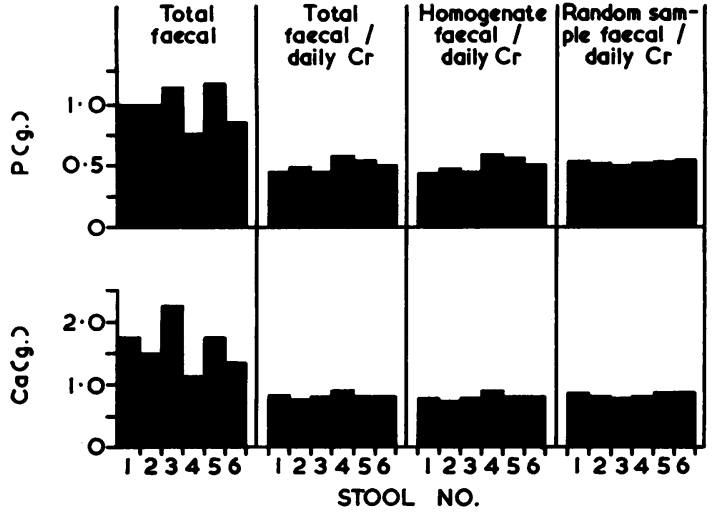

FIG. 6. From each stool a random sample was taken for analysis for calcium, phosphorus, and chromium. A larger sample was analysed after homogenization, and the remainder also analysed after homogenization. Total results on each stool were then obtained by appropriate additions. Only six stools were passed by this constipated patient in 12 days despite the liberal use of purgatives. No other therapy was given throughout.

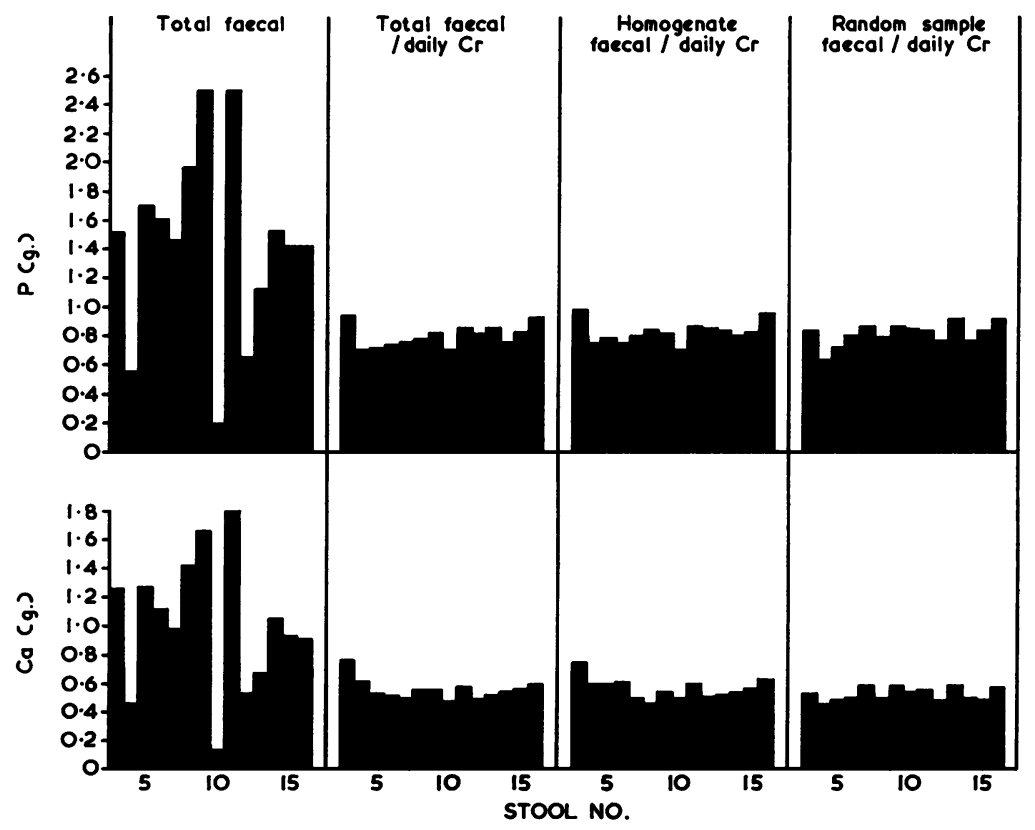

FIG. 7. The stools were treated substantially as is described in the legend to Figure 6. This patient passed 16 stools in 30 days. 
calcium intake of $3 \mathrm{~g}$. a day, as recommended by some for osteoporosis (see Lancet, 1963.1,538), the error would be $210 \mathrm{mg}$. a day, giving false hopes of a cure.

Figure 3 shows that while the majority of the sixday faecal collections contained a weight of chromium reasonably near to the six-day intake, some six-day collections contained as little as a two-day intake, and others the equivalent of up to an 11-day intake. In some cases (as in no. 8) such large variations could have been anticipated because of constipated, infrequent, and irregular bowel actions, but in other cases surprising variations occasionally occurred despite regular daily bowel actions with well-formed stools in which the carmine markers were clearly visible. When such variations occurred, there were also irregularities in the faecal calcium, phosphorus, and magnesium levels (see Fig. 4) and these irregularities largely disappeared when results were corrected for chromium content.

It is concluded that while the carmine marking of stools gives nearly constant chromium content in a few patients, definite and significant variations occur in most patients and very large variations in a small number of patients.

Figure 5 shows how when individual stools were analysed for calcium and chromium, the two elements apparently followed one another remarkably closely so that when calcium in each stool is expressed per daily chromium intake, there is remarkably little variation from stool to stool. Agreement is so close in fact that a small rise in faecal calcium following daily intravenous calcium infusions can be readily identified. Similarly, the speed of the effect of vitamin D on faecal calcium is readily observed. It is therefore concluded, first, that any one stool analysed for calcium and chromium will give an accurate indication of the daily faecal calcium, and secondly, that the accidental loss of a complete stool during a 'six-day' collection would be precisely indicated by a corresponding fall in chromium, the system of correcting calcium results for chromium recovery being a valid one.

Two other patients were studied in a similar way but in greater detail. The results in patient Alfred R. are shown in Figure 6. It is apparent that the calcium and phosphorus have followed the chromium so closely that consistent results are obtained from analysis of a small random sample of each stool. The sample need not even have been weighed, but merely ashed and analysed for calcium, phosphorus, and chromium. Inspection of Fig. 6 shows the remarkable fact that this procedure gave even more consistent results than analysis on homogenized remainders of stools. This must mean that the stools were homogeneous before treatment in the homogenizer, and that the homogenization procedure was in fact a centrifugal separation.

The results in patient E.McC. shown in Fig. 7 were slightly different. First, the results, corrected for chromium, were not quite as consistent from day to day, either in the random samples of stools or in analyses of whole stools. Secondly, the day-to-day variations were smaller in the analyses of whole stools than in the random sample analyses. Nevertheless, the analysis of a random sample of stool for calcium and chromium seems to have provided a way of calculating the daily faecal calcium to within $100 \mathrm{mg}$. While occasional analysis of a random sample of stool for chromium, calcium, and phosphorus would provide a very rapid and laboursaving method of calculating faecal calcium and phosphorus, it is not suggested here that this is the technique of choice for accurate balance work. For such work it still seems desirable to collect all the stools and pool several days' stools. It is strongly suggested on the other hand, that the loss of stools, whether on toilet paper, or in manipulation, or from accidental rejection of a stool, will lead to no significant error provided the results are corrected for the chromium content of the stools.

Apart from the gain in accuracy and consistency using the continuous chromium marking technique, other advantages can be derived. First, the six-day balance period used here could be shortened to a four-day period without significant loss of accuracy. Since it has been our practice generally to carry out two or three six-day balance periods before therapy, each patient can now be saved four to 10 days of balance study. Secondly, when it is desired to measure the faecal content of a substance which is not dependent upon intake, this can be done without a metabolic unit and indeed on an out-patient basis. A patient need only be given the chromium sesquioxide capsules for a few days and told to bring any single convenient stool at any time after the second day. This can then be homogenized and analysed for chromium and the appropriate substance under investigation. We have found this technique to be useful for measuring faecal lead in a patient with lead poisoning who was investigated and treated in a non-metabolic ward. Faecal fat would also seem to be readily measureable by use of a chromium sesquioxide marker provided that the chromium follows the fat sufficiently closely. This problem is under investigation at the present time.

Individual stools or fractions of stools were not analysed for magnesium and therefore it can neither be claimed nor denied that the chromium follows magnesium as closely as calcium. Since both calcium and magnesium are largely insoluble in the stools, however, it seems reasonable to suppose that 
the chromium does follow the magnesium sufficiently closely for present purposes. When in fact the "sixday' faecal magnesium results were corrected for chromium content, the results became very much more consistent as is shown in Figure 4.

The additional labour required for the analysis of the chromium in stools by present techniques is remarkably small. The rewards on the other hand are very great, with saving of time of investigation, with greatly improved accuracy and consistency of results, and with increased confidence in the results. The main disadvantage has been the labour of accurately filling the chromium sesquioxide capsules by hand. (Messrs. Sandoz Products Ltd. of Horsforth, Yorkshire, have now kindly agreed to undertake this for us with the aid of appropriate machinery, thus removing this main disadvantage.) It is intended in this unit to continue indefinitely with the combined interrupted carmine red and continuous chromium marking of stools for all calcium balance work. Further, it is suggested that this technique should be considered essential for all calcium balance work when a high degree of accuracy is required. Such accuracy is required, especially when the intake of calcium is high.

\section{SUMMARY}

Results of continuous chromium sesquioxide and interrupted carmine red marking of faeces are reported on the first 103 six-day balance periods. The errors of the carmine marking method are exposed. Using a correction factor based upon the chromium recovery in the 'six-day' faecal collections, the accuracy and consistency of faecal calcium, magnesium, and phosphorus results are greatly improved.

In three cases, each individual stool was analysed for calcium, phosphorus, and chromium. The daily faecal calcium and phosphorus results calculated from each stool were reasonably consistent from stool to stool. In two cases random samples of each stool were analysed for calcium, phosphorus, and chromium. In one of these cases, the calculated daily calcium and phosphorus levels were extremely consistent from day to day: in the other case consistency was not quite as impressive, but still good.

The advantages of continuous chromium sesquioxide marking are considerable and are stressed. The additional labour can be made very small.

It is a pleasure to be able to acknowledge the valuable assistance given by Sister Walker, Sister Orson, and the nursing and dietetic staff of the metabolic unit. The flame photometric work was carried out by Mr. D. Newton, and the phosphorus and nitrogen determinations by Miss V. Grayson. I am grateful to Dr. F. W. Heaton for the magnesium determinations. Mr. Blacow, the Infirmary pharmacist, kindly arranged for the handfilling of the chromium sesquioxide capsules.

\section{REFERENCES}

Anderson, J., and Weinbren, I. (1961). The determination of chromium and calcium in the faeces by flame spectrophotometer. Clin. chim. Acta, 6, 648-651.

Dawson, J. B., and Heaton, F. W. (1961). The determination of magnesium in biological materials by atomic absorption spectrophotometry. Biochem. J., 80, 99-106.

Fiske, C. H., and Subbarow, Y. (1925). The colorimetric determination of phosphorus. J. biol. Chem., 66, 375-400.

Humphreys, E. R., and Scott, P. P. (1962). Observations on the use of markers in determining food transit times in the cat. J. Physiol. (Lond.), 162, 28-29P.

MacIntyre, I. (1957). The flame-spectrophotometric determination of calcium in biological fluids and on isotopic analysis of the errors in the Kramer-Tisdall procedure. Biochem. J., 67, 164-172.

Reifenstein, E. C., Albright, F., and Wells, S. L. (1945). The accumulation, interpretation, and presentation of data pertaining to metabolic balances, notably those of calcium, phosphorus and nitrogen. J. clin. Endocr., 5, 367-395.

Whitby, L. G., and Lang, D. (1960). Experience with the chromic oxide method of fecal marking in metabolic balance investigations on humans. J. clin. Invest., 39, 854-863.

Wiggins, H. S., and Dawson, A. M. (1961). An evaluation of unabsorbable markers in the study of fat absorption. Gut, 2 , 373-376. 